\title{
Proceedings of the 148th Semon Club, 17 November 2014, ENT Department, Guy's and St Thomas' NHS Foundation Trust, London, UK
}

\author{
Chairperson: Miss Elfy B Chevretton, Guy's and St Thomas' NHS Foundation Trust \\ Secretary: Mr Sherif Haikel, Royal National Throat, Nose and Ear Hospital, University College London Hospitals NHS Trust \\ Invited panel for pathology: Dr Ann Sandison, Charing Cross Hospital, Imperial College Healthcare NHS Trust \\ Invited panel for radiology: Dr Steve Connor and Dr Ata Siddiqui, Guy's and St Thomas' NHS Foundation Trust \\ Professor Leslie Michaels prize for the best presentation of the meeting was awarded to Emma Gosnell for "“I can't swallow doctor" - an \\ unusual cause of dysphagia'.
}

The chairperson and secretary have edited the proceedings of the meeting to reflect the discussion of each case by the expert panel and audience during the Semon Club meeting.

\author{
Head and neck section \\ Chairperson: Mr Richard Oakley
}

'I can't swallow doctor' - an unusual cause of dysphagia

E J Gosnell, S Agrawal, V L Sharma

From the Fairfield General Hospital, Bury

\section{Introduction}

We present a case of dysphagia in a 35-year-old man subsequently diagnosed with a retropharyngeal cavernous lymphangioma. Lymphangiomas are malformations of the lymphatic system. Although found at all ages, retropharyngeal lymphangioma is not commonly seen in adults.

\section{Case report}

A 35-year-old man presented with a 3-month history of progressive dysphagia and hoarseness. His medical history included depression, cigarette smoking and high alcohol intake. Nasendoscopy identified an upper pharyngeal wall swelling. A computed tomography scan of the neck, panendoscopy and ultrasound-guided biopsy were arranged. The latter did not provide a tissue diagnosis. An external approach excision biopsy was subsequently performed.

\section{Radiology}

Computed tomography scanning identified a large, bi-lobed soft tissue mass in the retropharyngeal region. The lesion displaced the larynx and hypopharynx; however, no lymphadenopathy or aggressive features were seen. Subsequent magnetic resonance imaging confirmed a likely submucosal mass measuring $10 \mathrm{~cm}$ in craniocaudal dimension.

\section{Histology}

Histology confirmed a multi-cystic, encapsulated brown lesion measuring $92 \times 55 \times 50 \mathrm{~mm}$, with dilated anastomosing vascular channels. The lumens showed proteinaceous fluid with lymphocytes. The overall features were consistent with a cavernous lymphangioma, with no evidence of malignancy.

\section{Management}

As initial biopsies did not provide a tissue diagnosis, subsequent management was discussed by the head and neck multidisciplinary team. Given concerns about the patient's airway, pharyngoscopy and an external approach excision biopsy were performed. Histology confirmed a completely excised cavernous lymphangioma.

\section{Discussion}

Our differential diagnoses had included lymphoma and soft tissue tumours. Mr Oakley enquired whether a core biopsy would have been adequate to exclude malignancy. Dr Sandison said that this may not have been representative and that formal excision biopsy was necessary for an accurate diagnosis. Mr Oakley remarked that this is a very rare diagnosis in adults. Dr Sandison had previously seen one case, in an 18 year old. She postulated that perhaps these lesions are present from birth but sometimes present later in life.

An unusual mass which brought a tear to his eye

S Bickerton, R J Glore, S Winter

From the Churchill Hospital, Oxford

Introduction

Lacrimal sac tumours are rare neoplasms that may present as a mass, epiphora or recurrent dacryocystitis. The most common types are papilloma, primarily in the form of a benign epithelial tumour; however, rarely, a non-epithelial oncocytic adenocarcinoma can occur. Here we report a case of a primary nasolacrimal adenocarcinoma with oncocytic change.

\section{Case report}

A 68-year-old male presented with a 4-month history of a painless, progressive, irregular $3 \mathrm{~cm}$ mass in his right lower eyelid, associated with epiphora.

\section{Radiological findings}

A computed tomography scan of the orbits showed a homogeneous mass, with irregular margins, centred on the right 
medial canthus and lacrimal sac. The mass extended into the retroseptal extraconal orbit, abutting the right globe. Dr Connor reminded the audience of the importance of excluding bony erosion or soft tissue in the inferior meatus, as these tumours can spread down the nasolacrimal duct.

\section{Histological findings}

Biopsied tissue was extensively infiltrated by carcinoma composed of oncocytic cells, with moderate nuclear pleomorphism and mitoses. Immunohistochemical staining showed positivity for pan-cytokeratin, cytokeratin 7, epithelial membrane antigen, gross cystic disease fluid protein 15 and androgen receptor.

\section{Management}

The patient underwent primary excision of the nasolacrimal duct tumour, right orbital exenteration with partial maxillectomy, a right neck dissection, and a thoracodorsal artery perforator flap reconstruction, followed by adjuvant radiotherapy.

\section{Discussion}

Dr Sandison pointed out that expression of receptors in common with breast cancer, such as gross cystic disease fluid protein 15 and human epidermal growth factor 2, has led to research investigating whether breast cancer therapies such as trastuzumab may be beneficial.

\section{Conclusion}

Nasolacrimal duct tumours are rare and frequently invasive. They require early diagnosis and aggressive management. Receptor-targeted therapy may have a role in future management.

\section{What a pain in the neck}

\section{G H Jones, D Thorley, S Penney}

From the Tameside Hospital NHS Foundation Trust, Ashton under Lyne

\section{Case report}

A 21-year-old female presented to the head and neck clinic with a 6-week history of a painful anterior midline neck swelling. She was constitutionally well, with no past medical history of note. She was admitted for empirical intravenous antibiotics after an ultrasound-guided aspiration drained $8 \mathrm{ml}$ of turbid fluid. During this time, she became pyrexial with raised inflammatory blood markers. Two days later, an incision and drainage revealed a combination of pus and lymphoid tissue.

\section{Histological findings}

Fine needle aspirates and initial histology demonstrated an inflammatory process, with microbiology cultures repeatedly negative. Test results of an atypical infection screening that included tests for human immunodeficiency virus, Epstein-Barr virus, hepatitis, bartonella, actinomycosis and tuberculosis were also negative.

\section{Radiological findings}

A computed tomography scan confirmed an anterior, mainly cystic lesion (measuring $57 \mathrm{~mm}$ craniocaudally), located just above the sternal notch, with bilateral lymphadenopathy. Magnetic resonance imaging revealed the abscess in possible continuity with a thyroglossal tract and cyst, postulating the diagnosis of infected thyroglossal duct cyst.

\section{Management and diagnosis}

A modified Sistrunk procedure was performed, with excision of the abscess and thyroglossal tract. Final histology confirmed the diagnosis of infected thyroglossal duct cyst, but also revealed a surprise finding of Hodgkin's lymphoma.

\section{Discussion and lessons learned}

The case was well managed given the significant diagnostic challenges faced. Perseverance is important when the diagnosis is elusive.

\section{Conclusion}

It is important to use a multidisciplinary approach in diagnostically challenging cases. Collaboration with the histopathologist and radiologist is essential to ensure information is shared; this may reveal the diagnosis or help target investigations more effectively. Mr Oakley noted that lymphomas related to thyroglossal cysts are usually seen in young patients.

The great mimicker: a rare case of head and neck lump in the presence of human immunodeficiency virus

H Ramotar, L Cheung, L Pitkin

From the Frimley Park Hospital NHS Foundation Trust

\section{Introduction}

Inflammatory pseudotumours in the head and neck are rarely reported in patients with human immunodeficiency virus (HIV) positivity. These lesions, which have many names in the literature, including plasma cell granuloma, pose a diagnostic dilemma.

\section{Case report}

We present a case of inflammatory pseudotumour in the left tonsil, with cervical lymphadenopathy, in a 49-year-old, HIV-positive, female patient.

\section{Radiological findings}

Neck and thorax contrast-enhanced computed tomography and neck magnetic resonance imaging with gadolinium showed an enhancing mass in the left tonsil involving the left vallecula and pyriform sinus, with probable bilateral cervical nodal metastases. These scans suggested an invasive cancer with metastases.

\section{Histological findings}

Immunohistochemistry of tonsil and lymph node tissues confirmed a polyclonal population of plasma cells. Tissue structures were obliterated by a densely sclerotic fibro-inflammatory process. Stains for micro-organisms, and markers of infection and disease processes, were negative. No malignancy was identified, which was not in keeping with the clinical picture. A second opinion was sought from a tertiary centre; six months later, the provisional diagnosis of inflammatory pseudotumour was confirmed.

\section{Management}

A left tonsillectomy with a wide local excision was initially performed. The cervical lymph node was excised later when it enlarged. The patient's symptoms resolved completely. She healed well, and was discharged.

\section{Discussion}

Delay in diagnosing an inflammatory pseudotumour may lead to unnecessary surgery. Mr Oakley suggested that as the lesions were anatomically separate, an immunoglobulin G4 related sclerosing disease (e.g. Kuttner's tumour) was a 
possible differential diagnosis. If after initial investigations no conclusions are reached, Dr Sandison recommended autoimmune testing.

\section{Could it be cancer?}

R Ghedia, J Weir, A Mace

From the Charing Cross Hospital, London

\section{Introduction}

Nodular fasciitis is a benign, idiopathic, reactive proliferation of myofibroblasts found in the subcutaneous fascia.

\section{Case report}

A 74-year-old Asian male presented with a 3-month history of a right-sided cheek lump. Initial ultrasound scans and fine needle aspiration (FNA) results were inconclusive, and so the multidisciplinary team decision was for further imaging and subsequent excision.

\section{Radiological findings}

Ultrasound scans pointed towards a pleomorphic adenoma, but FNA findings suggested a necrotising malignancy with squamous differentiation. Subsequent magnetic resonance imaging showed a well-defined focus, possibly invading part of the masseter. A positron emission tomography scan showed no other suspicious lesions.

\section{Histological findings}

The lesion was excised and found to be a non-encapsulated lesion composed of spindle cells, with no atypia. There was no local invasion. Immunohistological staining showed that the lesion was strongly positive for smooth muscle antibody and weakly positive for cyclin D1. It was negative for beta-catenin, calponin, S100, ALK-1, desmin, epithelial membrane antigen and cluster of differentiation 34. Overall, it was confirmed to be nodular fasciitis.

\section{Management}

In light of inconclusive imaging and cytology findings, excision biopsy was recommended. Nodular fasciitis rarely recurs after surgical excision and the prognosis is excellent. The patient was therefore reassured and discharged.

\section{Discussion and conclusion}

Dr Sandison emphasised that nodular fasciitis is an important differential diagnosis to consider when treating patients with soft tissue tumours as it can easily be misdiagnosed as sarcoma, both clinically and histologically, because of the cellularity, mitotic figures and rapid growth. Dr Siddiqui agreed that further imaging is essential when primary investigations are inconclusive to help pre-operative planning and decision making, but ultimately a tissue diagnosis is desirable.

\section{Rhinology section}

Chairperson: Mr Hari Jeyarajan

\author{
Recovery of total anosmia following septorhinoplasty \\ L Ritchie, N A Watson, P Andrews \\ From the Royal National Throat, Nose and Ear Hospital, University \\ College London Hospitals NHS Trust
}

\section{Introduction}

Anosmia affects 5 per cent of the normal population. The role of nasal surgery in its treatment remains unclear. The three commonest causes for anosmia are head injury, upper respiratory tract infection, and nasal or sinus disease. The pathogenesis of smell dysfunction, the least known-about sense disorder, can be divided into conductive and sensorineural causes.

\section{Case report}

A 61-year-old male underwent external septorhinoplasty to improve his nasal airflow. He had fractured his nose with a cricket ball 40 years previously. Prior to his injury, he had been a sommelier. His injury was associated with total anosmia. After surgery performed to restore his nasal airflow, his nasal function and quality of life scores improved, with an unexpected post-operative recovery of his sense of smell, which was corroborated by a high score in the University of Pennsylvania Smell Identification Test ('UPSIT').

\section{Radiological findings}

Coronal computed tomography of the paranasal sinuses showed marked septal deviation to the right. In addition, there was hypertrophy of the left inferior turbinate and a right-sided concha bullosa.

\section{Management}

Open approach septorhinoplasty was carried out via an 'inverted-V' incision. The nasal bones were fractured and re-positioned, and the dorsal hump was reduced. Septal cartilage was harvested, and spreader grafts and an external nasal valve strut were inserted. A plaster of Paris cast and splints were placed.

\section{Discussion}

In light of the dramatic olfactory improvement, it is arguable there was both a conductive and sensorineural component to the patient's anosmia. The panel agreed that little is known about the pathophysiology of anosmia and thus it warrants further research.

\section{Pitfalls of recurrent epistaxis associated with septal perforation}

\section{Tornari, L Ritchie, P Andrews}

From the Royal National Throat, Nose and Ear Hospital, University College London Hospitals NHS Trust

\section{Introduction}

Epistaxis and septal perforation are common ENT presentations which prompt investigation when they coexist or are associated with other problems. This clinical picture suggests the possibility of granulomatosis with polyangiitis (formerly Wegener's granulomatosis), which is investigated by serology for cytoplasmic or perinuclear antineutrophil cytoplasmic antibodies (ANCAs). However, one in five patients with active limited disease has negative ANCA screen results.

\section{Case reports}

We report on 2 male patients, aged 66 and 40 years, who underwent multiple ENT procedures for troublesome epistaxis and large nasal septal perforations. The older patient had suffered symptoms from childhood; the younger patient also had an indolent onset of symptoms. The older patient underwent sphenopalatine artery ligation, embolisation and anterior ethmoid ligation for recurrent epistaxis. 
The younger patient had Silastic ${ }^{\circledR}$ splints inserted and subsequent reconstructive septorhinoplasty using a rib graft to prevent nasal crusting and improve the nasal airway. Though granulomatosis with polyangiitis was suspected, both patients' initial serology results revealed no abnormalities.

\section{Radiological findings}

Computed tomography demonstrated nasal septal inflammation in the oldest patient. Dr Connor also pointed out wide defects into both maxillary antra and erosion of the turbinates. He reminded the audience that some cases progress to autorhinectomy.

\section{Histological findings}

Intra-operative biopsies revealed granulomatous inflammation in both patients.

\section{Management}

The histological findings prompted rheumatology referrals. Both patients subsequently seroconverted to cytoplasmic ANCA positivity. Cyclophosphamide chemotherapy led to improvement in their nasal and systemic symptoms.

\section{Discussion}

Dr Sandison noted that she had never confirmed a granulomatosis with polyangiitis diagnosis on histology alone, as the changes were not specific to granulomatosis with polyangiitis.

\section{Conclusion}

Patients with recurrent epistaxis in the context of nasal septal perforation need thorough investigation for possible granulomatosis with polyangiitis, and ENT surgeons should be aware that negative ANCA screens do not exclude this diagnosis.

Conductive hearing loss as a presentation of a cocaineinduced midline destructive lesion

\section{K Steele, D Tighe, S T Baer}

From the Conquest Hospital and Eastbourne District General Hospital, East Sussex Healthcare NHS Trust

\section{Case report}

A 57-year-old man presented with a 5-month history of hearing loss and 2 weeks of regurgitating liquid through his nose. He admitted to occasional cocaine use. He was found to have a $5 \mathrm{~mm}$ defect in his soft palate, bilateral middle-ear effusions with a corresponding conductive hearing loss, and massive destruction of the mid-nose on endoscopic examination.

\section{Radiological findings}

Computed tomography and magnetic resonance imaging highlighted an absent septum, dehiscence in the soft palate, and mucosal thickening of the maxillary and ethmoidal sinuses.

\section{Histological findings}

Biopsies revealed a mixed inflammatory cell infiltrate with abundant granulation tissue and avascular necrosis of a septal cartilage fragment.

\section{Management}

A diagnosis of cocaine-induced midline destructive lesion was made. This condition is managed conservatively, focusing on abstinence from cocaine to halt the destruction. For this patient, a palatal obturator prevented oronasal regurgitation. His hearing returned to normal limits following bilateral grommet insertion.

\section{Discussion and learning points}

Dehiscence in the palate affects the mechanism of tensor palatini muscles on the distal eustachian tube. The subsequent eustachian tube dysfunction, causing negative middle-ear pressure and middle-ear effusion, is a likely explanation for the patient's hearing loss.

The differential diagnoses for nasal destruction cases include small vessel vasculitides, polychondritis and granulomatous disorders, as illustrated by the previous case presented at the meeting. Mr Haikel enquired if recurrent trauma and chronic infection could have caused the same histological changes in both this case of cocaine-induced midline destructive lesion and the previous case of granulomatosis with polyangiitis. Dr Sandison felt that if that were the case, she would have expected a higher proportion of neutrophils and fibroblasts, with a slightly different histological picture.

Nasal obstruction in a patient with a history of a vascular skull base tumour

R Edmiston, P Pal, R K Bhalla

From the Tameside Hospital NHS Foundation Trust, Ashton under Lyne

\section{Introduction}

Solitary fibrous tumours are extremely rare mesenchymal tumours, and bleeding can often complicate surgery. This case describes treatment by an endoscopic approach, using novel operative strategies in a high-risk patient who was unable to have a blood transfusion.

\section{Case report}

A 28-year-old Caucasian female had undergone transnasal surgery 8 years previously for a right-sided sinonasal solitary fibrous tumour. She re-presented on this occasion with a recurrence of symptoms, but peri-operative embolisation was found to be too hazardous.

\section{Radiological findings}

Dr Connor demonstrated a partially calcified mass centred around the superior meatus, with involvement of the right cribriform plate and intracranial extension.

\section{Histological findings}

Histopathological assessment revealed a spindle cell neoplasm composed of densely packed fascicles, sometimes wavy in appearance. Dr Sandison demonstrated 'staghorn antler' vessels on haematoxylin and eosin stain.

\section{Management}

An extended transnasal approach was undertaken with the use of image guidance, a microdebrider and a coblator. The lesion was completely excised, with clear margins laterally, superiorly and posteriorly. There was no evidence of recurrence at 12-months' follow up.

\section{Discussion}

Mr Jeyarajan felt that the endoscopic approach had better functional outcomes, and was the same as open surgery in terms of disease control.

The use of coblation was debated, with suggestions for consideration including the use of a Helica thermal coagulator as an alternative haemostatic adjunct. 


\section{Conclusions}

Coblation was found to be very useful in assisting in the excision of this vascular skull base tumour in a case complicated by the patient's status as a Jehovah's Witness.

\section{A curious case of nasal obstruction and pain \\ R Edmiston, Y Karagama \\ From the Manchester Royal Infirmary}

\section{Introduction}

Nasal sarcoidosis is very uncommon, affecting less than 10 per cent of sarcoid sufferers. This, in combination with the pathological mechanism of the disease, makes it notoriously difficult to manage. Treatments vary from corticosteroids, methotrexate and azathioprine, to nasal surgery, but often the results are poor.

\section{Case report}

A 51-year-old African lady was referred to ENT with persistent nasal obstruction and nasal pain. Examination revealed granulomatous submucosal deposits throughout the nasal vestibule and loss of nasal tip support.

\section{Radiology}

Imaging revealed classical cobble stoning of her nasal mucosa; however, no sinus involvement was found, with bilateral patent ostiomeatal complexes.

\section{Histology}

Large and florid epithelioid giant cell granulomas were found on nasal biopsy, with no evidence of necrosis, and a variable lymphoid infiltrate thought to be consistent with sarcoidosis.

\section{Management}

Both systemic and topical steroids failed to improve her nasal airway, and an operative technique was therefore adopted. The patient underwent examination under anaesthetic of the nose and excision of the right vestibular lesion. Despite one post-operative episode of self-limiting epistaxis, this has proved a suitable treatment modality for her nasal pain.

\section{Discussion}

Dr Connor identified radiological changes not initially reported, namely bony erosion of the hard palate, alveolus and nasal bones, and thickening of the anterior septum, probably as a result of the sarcoidosis.

It was accepted that there is limited evidence for the management of such painful lesions. The use of functional endoscopic sinus surgery in sarcoidosis patients was discussed.

\section{Conclusions}

Surgery may have a significant role in the treatment of localised painful sarcoid lesions, but research is lacking because of the rare nature of the disease.

\section{Otology section}

Chairperson: Mr Rupert Obholzer

Difficult to find: an unusual case of unilateral tinnitus

V Harries, S Cartwright, N Saunders

From the Royal Sussex County Hospital, Brighton

\section{Introduction}

Tumours of the middle ear are a rare entity and the differential diagnoses are varied. We detail a case that highlights some of the considerations in management.

\section{Case report}

A 38-year-old lady was referred to the ENT department with left-sided pulsatile tinnitus and hearing loss. On examination, she had an intact tympanic membrane with features of a pale grey antero-superior middle-ear lesion and effusion.

\section{Radiological findings}

A magnetic resonance imaging scan revealed non-specific retained secretions filling the middle-ear cleft. A computed tomography scan showed no erosion of the tegmen, scutum or jugular bulb.

\section{Histological findings}

Irregularly arranged glial cells with immunoreactivity for glial fibrillary acidic protein and S100 markers were identified. The features were in keeping with glioneuronal tissue.

\section{Management}

The patient underwent tympanotomy and excision of the middle-ear lesion. Intra-operatively, an encephalocele was suspected, as clear fluid was found within the middle ear. However, the fluid tested conclusively negative for glucose on dipstick analysis, and on further review of the imaging no evidence of an encephalocele could be seen. Postoperatively, the patient developed a confirmed cerebrospinal fluid (CSF) leak.

\section{Discussion}

The case was discussed at the Semon Club meeting, where the radiological images were reviewed. Dr Siddiqui identified bone scalloping along the floor of the middle cranial fossa, with evidence of brain herniation, confirming an encephalocele.

\section{Conclusion}

This case highlights the difficulties in identifying an encephalocele and illustrates that a negative result for glucose on dipstick analysis does not rule out CSF. This patient will undergo a further operation to repair the CSF leak.

An unusual cause of a hoarse voice, facial weakness and dysphagia

R Sprout, M Bull, N Bleach

From the Frimley Health NHS Foundation Trust

\section{Introduction}

Granulomatosis with polyangiitis most commonly presents with respiratory or renal involvement. However, neurological manifestations are becoming increasingly recognised. We present a case to highlight these sequelae in otolaryngology.

\section{Case report}

We report the case of a 64-year-old female who presented with right vocal fold palsy followed by right facial nerve palsy.

\section{Radiological findings}

Post-contrast magnetic resonance imaging scans of the brain displayed an enhancing mass in the right cerebellopontine angle, and meningeal thickening and enhancement. The patient tested positive for cytoplasmic antineutrophil 
cytoplasmic antibodies, with high proteinase-3, consistent with granulomatosis with polyangiitis.

\section{Histological findings}

Biopsy of the right cerebellopontine angle lesion revealed chronic inflammatory changes consistent with meningeal inflammation associated with granulomatosis with polyangiitis.

\section{Discussion}

Presentation of granulomatosis with polyangiitis with neurological manifestations is rare but is becoming increasingly recognised. Less than 40 cases of meningeal involvement in granulomatosis with polyangiitis have been described. Dysphagia as a presenting feature of granulomatosis with polyangiitis is uncommon, having only been described three times previously. However, vocal fold palsy, dysphagia and facial nerve palsy are common presentations to the otolaryngologist, and as such this case demonstrates the importance of thorough history taking and the consideration of a wide range of potential differential diagnoses when investigating such patients.

\section{Conclusions}

Granulomatosis with polyangiitis can manifest in any system, and testing should be considered whenever symptoms are unexplained or atypical.

Recurrent seizures, headaches and bilateral hearing loss without otogenic cause

W Yeung, G Chawdhary, R Corbridge

From the Royal Berkshire Hospital, Reading

\section{Introduction}

Central skull base osteomyelitis is a rare infection, typically arising from medial spread of necrotising otitis externa. Commonly presenting as non-specific headache and facial pain, diagnosis can be difficult and imaging may mimic malignancy. We present a case of central skull base osteomyelitis in the absence of otogenic or any other source of infection.

\section{Case report}

An 80-year-old man with Crohn's disease presented following a tonic-clonic seizure on a 3-month background of right parietal headache. There was no history of otitis externa. Neurological and ENT examination findings were unremarkable. Whilst awaiting urgent tissue diagnosis, he re-presented with seizure and new bilateral deafness.

\section{Radiological findings}

Gadolinium-enhanced magnetic resonance imaging (MRI) revealed a submucosal nasopharyngeal soft tissue mass. This mass, which extended posteriorly to the foramen magnum, abutting the right internal carotid, raised suspicion of a metastatic deposit. Repeat MRI at second presentation showed lesion progression, with bilateral jugular foramen and internal carotid involvement. A new rimenhancing collection was noted, along with abnormal enhancement seen throughout the clivus. Tissue obtained via a computed tomography (CT) guided biopsy was analysed.

\section{Histological findings}

Histological appearances of the biopsy tissue were in keeping with an abscess. Microbiology cultures grew Pseudomonas aeruginosa.

\section{Management}

The patient was treated with intravenous ceftazidime (for six weeks) and oral ciprofloxacin (for six weeks). His headache and hearing loss completely resolved, with normalisation of inflammatory markers. Post-treatment CT demonstrated a marked reduction in the size and extent of the mass. He remains under long-term follow up.

\section{Discussion}

Central skull base osteomyelitis should be considered in cases of an unexplained nasopharyngeal soft tissue mass, even in the absence of ear or lateral skull base infection. Prompt tissue diagnosis enables timely antibiotic treatment.

\section{Persistent ear discharge following treatment for tuberculosis mastoiditis}

C Tornari, C Huins, J Lavy

From the Royal National Throat, Nose and Ear Hospital, University College London Hospitals NHS Trust

\section{Introduction}

A 12-year-old girl was referred to ENT because of left ear bleeding and pain. She had been treated with chemotherapy at the age of one month for tuberculosis (TB) in this ear. Previous investigation had revealed apparent total ossification of the left middle ear and associated profound mixed hearing loss.

Case report

Clinical examination revealed a featureless left tympanic membrane with no active infection, and mild left cervical lymphadenopathy. Recent inflammatory polyp excision from this ear revealed simple granulation.

\section{Radiological findings}

Magnetic resonance imaging (MRI) and computed tomography examination demonstrated a bony defect in the roof of the left external auditory canal associated with a mastoid mass that was consistent with cholesteatoma.

\section{Histological findings}

Histology demonstrated fibrotic and chronically inflamed tissue with a histiocytic response to keratin flakes, consistent with chronic otitis media and cholesteatoma.

\section{Management}

A limited mastoidectomy was performed because of a high and anterior sigmoid sinus. The cholesteatoma extended medial to this structure and was removed. The external auditory canal defect that had been created by the initial TB infection was repaired with conchal cartilage and bone graft. The patient will be followed up by routine non-echo planar imaging, diffusion-weighted MRI scanning.

\section{Discussion}

The above clinical picture was novel to the attendees, demonstrating the rarity of this complication in the UK.

\section{Conclusion}

This case was unusual in that the mastoid cholesteatoma had arisen from an external auditory canal defect which was caused by TB. Otologists should, therefore, be aware of occult sources of cholesteatoma in such patients. 
Cholesteatoma by any other name?

A Testera, H Jeyarajan, R Obholzer

From the Guy's and St Thomas' Hospital NHS Foundation Trust, London

\section{Case report}

A 29-year-old male with a long history of generalised headaches had presented with left-sided facial nerve palsy. He had no history of ear disease. He had been started on a course of oral steroids, with the presumptive diagnosis of Bell's palsy. When he failed to respond appropriately, he was referred to our service. On examination, he had findings consistent with House-Brackmann grade IV palsy. General examination findings were otherwise unremarkable, with normal otoscopy findings and no other cranial nerve palsies.

\section{Radiology}

A non-contrast computed tomography scan revealed an incidental lytic lesion in the left occipital bone. There were no other significant findings. Subsequent magnetic resonance imaging with no contrast enhancement showed the occipital lesion to be hyperintense.

\section{Pathology}

Ultrasound-guided fine needle aspiration was performed to clarify the diagnosis. The abundance of keratin confirmed an intradiploic epidermoid.

\section{Management}

The patient was counselled regarding the nature of the lesion and the tumour was electively excised. His Bell's palsy completely resolved.

\section{Discussion}

Epidermoid tumours represent $0.2-1.8$ per cent of all intracranial tumours. Intradiploic lesions account for 25 per cent of these tumours. Whilst it is possible to find these lesions throughout the cranial vault, they are most prevalent at the cerebellopontine angle (40-50 per cent) or the parasellar region (30 per cent).

Being generally asymptomatic and only slowly expansile, these lesions are often an incidental finding. Malignant degeneration is a rare but significant complication, with 58 cases reported in the world literature. It universally portends a dismal prognosis, with an average survival time of 12 months regardless of therapy.

\section{Paediatric section}

Chairperson: Mr Ian Hore

Rapidly expanding paranasal swelling in a child: third time lucky?

M Cherko, S Gane, R Hewitt

From the Great Ormond Street Hospital, London

\section{Introduction}

We report the interesting case of a two-year-old girl with a rapidly expanding left paranasal swelling, which eventually impaired left eye opening. Imaging showed a mass appearing to arise from the left superior alveolar ridge, extending lateral to the nose and inferior to the orbit. Core biopsies revealed a spindle cell tumour. Despite the benign nature of this tumour, she went on to have multiple operations and protracted treatment with multidisciplinary input.
Case report

Just four weeks after initial excision via a sublabial degloving approach, the patient developed a recurrent swelling and was taken back to the operating theatre for revision surgery with partial maxillectomy and reconstruction. Recurrence was again apparent six months later, this time with invasion into the left nasal cavity. Following a third excision and 52 weeks of chemotherapy, she now remains well.

\section{Radiological findings}

Magnetic resonance imaging showed a well circumscribed mass appearing to arise from the left superior alveolar ridge, just lateral to the nose and inferior to the left orbit.

\section{Histological findings}

Histology revealed a spindle cell tumour, which was not frankly malignant.

\section{Management}

Three excision operations and a reconstruction were performed. A 52-week protocol of vinblastine and methotrexate was administered.

\section{Discussion}

On reviewing the radiology, Dr Siddiqui highlighted the early erosive changes to the inferior orbital wall caused by this benign, expansile lesion. Dr Sandison confirmed a low-grade mesenchymal tumour with a proliferation rate below 5 per cent.

\section{Conclusion}

This case emphasises the difficulty in successfully treating even benign spindle cell tumours.

A persistent dry cough in an immunocompromised child

J Bates, A Hall, M Wyatt

From the Great Ormond Street Hospital, London

\section{Introduction}

We present the case of an emergency tracheostomy involving an underlying diagnosis not previously described in the paediatric literature.

\section{Case report}

An eight-year-old girl was referred to the Great Ormond Street Hospital immunology department from her local hospital with a year-long history of a dry cough. The patient had leucocyte adhesion deficiency, an extremely serious and rare form of congenital immunodeficiency that had previously been treated with a bone marrow transplant. The cough had worsened, with associated dysphonia and a feeling like 'something was stuck' in her throat.

On arrival at the Great Ormond Street Hospital, she had audible biphasic stridor, and when agitated her airway became more obstructed. Concerns were raised regarding possible extensive retropharyngeal lymphadenopathy given her underlying diagnosis. After appropriate imaging, she was taken to the operating theatre to investigate and secure her airway.

\section{Radiological findings}

Computed tomography findings demonstrated dramatic tracheal lumen obstruction at two levels: at the subglottis and at the sternal notch. These 'plaques' appeared to be low density and were inseparable from the tracheal wall. 


\section{Histological findings}

Tracheal specimens showed fragments of necrotic tissue and fibrin, with associated fungal spores and hyphae. Microbiology specimens grew Aspergillus fumigatus, which is commonly found in soil and compost. This is known to result in a classical 'silver-stained' appearance.

\section{Management}

Following tracheostomy, systemic anti-fungal therapy was given. Six weeks after the tracheostomy was placed, her airway had improved dramatically allowing decannulation.

\section{Conclusion and lessons learned}

A persistent dry cough in an adult immunocompromised patient should lead clinicians to consider invasive aspergillosis. The same considerations should be given to the paediatric population, with systemic anti-fungal treatment rather than reconstructive tracheal surgery recommended.

\section{Progressive 'Pinocchio' nasal deformity in a female adolescent}

B Silver, Y Bajaj, F K Seymour

From the Royal London Hospital, Barts Health NHS Trust

\section{Introduction}

'Pinocchio' nasal deformity is a rare but highly stigmatising condition that may lead to severe psychosocial problems. It usually results from soft tissue tumours such as superficial or deep haemangiomas, which often spontaneously regress early in life. In contrast, lymphatic malformations can evolve because of the influence of infection, trauma or hormonal changes. This results in destruction of lymphatic circulation and progressive expansion. These malformations require early surgical management to optimise cosmetic outcomes.

\section{Case report}

An adolescent female, who had been bullied by school peers, presented with a slow-growing (over a two-year period) bulbar lesion on the supratip of her nose. On examination, she had a soft, compressible, cystic mass on the nasal tip, which had a bluish hue. Anterior rhinoscopy findings were unremarkable.

\section{Radiological findings}

Magnetic resonance imaging showed a subcutaneous lesion, which was enhanced on T2-weighted imaging and measured $14 \times 9 \times 15 \mathrm{~mm}$. It was loculated and consistent with a lymphovenous malformation. There was no connection with the skull base.

\section{Histological findings}

Histology revealed fibro-fatty connective tissue containing a proliferation of vessels of varying sizes and dilated lymphatic channels which were confirmed with immunohistochemistry. Histology confirmed the clinical impression of a lymphovascular malformation.

\section{Management}

After counselling, the patient underwent excision of the lesion via an external septorhinoplasty approach. There was good post-operative cosmesis and patient satisfaction, with no recurrence after two years. This case highlights the importance of establishing the correct diagnosis in such cases, as an incorrect diagnosis may result in delayed surgery and worse cosmetic outcomes.

\section{Recurrent ear polyp in an adolescent}

B Silver, Y Bajaj, F K Seymour

From the Royal London Hospital, Barts Health NHS Trust

\section{Introduction}

Neurofibromas are benign tumours of the nerve sheath. Diffuse neurofibromas are an uncommon variant of neurofibromas that occur primarily in the head and neck area of young adults and children. It is an ill-defined infiltrative lesion that mainly affects the skin and subcutaneous tissues.

\section{Case report}

An adolescent female developed a non-painful soft tissue swelling that completely occluded the external auditory meatus. The swelling originated from the anterior cartilaginous canal, sparing the bony canal and tympanic membrane. There was no pain, bleeding or history of trauma. Audiometry findings were normal. Multiple attempts to excise this lesion were unsuccessful because of regrowth, which occurred within weeks. Development of an ipsilateral parotid swelling led to consideration of a possible congenital duplication of the external auditory canal or a first branchial cleft abnormality. The patient had no other neurofibromas or café-au-lait spots.

\section{Imaging}

A computed tomography scan revealed a superficial irregularity of the outer external auditory canal skin, with no bony erosion. The middle and inner ear were unremarkable. A magnetic resonance imaging scan revealed multiloculated areas of high T2 signal, consistent with a lymphovenous malformation. The lesion extended into the ipsilateral superficial parotid gland.

\section{Histology}

Dr Sandison demonstrated a vascular lesion with irregular islands of fibromyxoid tissue and a plexiform architecture. It was a benign lesion that had features of a hamartoma or vascular malformation. Immunohistochemistry suggested a neural origin.

\section{Management}

After multiple expert opinions, a diagnosis of diffuse neurofibroma was established. The patient has been referred to a quaternary centre for further management. This case presented a diagnostic challenge to all involved. Dr Sandison reminded the audience of the association with neurofibromatosis type 2 and the need for the patient to undergo genetic counselling.

\section{A case of stridor in a newborn}

B Patel, F Noonan, V Possamai

From the Evelina London Children's Hospital, Guy's and St Thomas' NHS Foundation Trust

\section{Introduction}

Stridor at birth is always pathological and warrants early referral for an ENT assessment prior to discharge from hospital. The differential diagnoses are wide, and include rare conditions that require a high index of suspicion and thorough investigation.

\section{Case report}

A healthy term baby boy was noted by his parents to have inspiratory stridor intermittently from birth. They sought 
medical advice following two cyanotic episodes in his first week at home. General examination and initial flexible nasendoscopy failed to detect an abnormality.

The patient underwent microlaryngoscopy. On the operating theatre table, with the shoulder roll in place and the head extended, a left lower anterior neck mass became apparent. Endoscopic examination confirmed laryngeal compression at the level of the neck mass, which accounted for the stridor.

\section{Radiological findings}

Magnetic resonance imaging of the neck showed a $2 \mathrm{~cm}$, well-defined unilocular cyst within the left lateral neck, causing displacement of the larynx.

\section{Management}

The patient underwent excision of the lesion via selective neck dissection, with identification and preservation of a tortuous left recurrent laryngeal nerve. The patient developed post-operative left vocal fold palsy, but went on to make a good vocal recovery by three months postoperatively.

\section{Histological findings}

Histology showed a benign thymic cyst. The cyst wall appeared to be fibrous, with no lining present. There was marked acute inflammation, accompanied by fibrovascular proliferation and fibrin deposition.

\section{Discussion}

After reviewing the radiology and histology, Dr Siddiqui and Dr Sandison felt that the features were consistent with an ectopic thymic cyst. 